authors' revised sequence of events also has some appeal, in that it ties ocean formation to the release of water-rich gas from massive, extended volcanic events. And, in the case of the ocean associated with the Arabia shoreline, it links ocean formation to the emergence of valley networks ${ }^{4}$ - abundant features on early Mars that carried water to the northern lowlands in which the oceans were situated.

Not surprisingly, the interplay of water and geodynamics over Mars's history is more complicated than presented in Citron and colleagues' analysis. During the period of interest, additional deformational processes occurred at local to global length scales that were not accounted for. Such processes are associated with, for example, mantle activity, impacts of asteroids or comets on to Mars's surface, glaciers, erosion and individual volcano growth, and could also have contributed to the deformation of the shorelines.

More-detailed mapping and modelling could clarify the contributions to the observed elevation changes and refine the sequence and relative timing of events. In addition, other, much shorter and more fragmented potential shorelines have been proposed ${ }^{14,15}$ that suggest the existence of seas and lakes, and that merit further study. Finally, the timing and role of volcanism, as it relates to the state and evolution of the atmosphere and to the persistence of water on the planetary surface, is far from understood. But, fortunately, the extensive database of remote and in situ observations of Mars continues to accumulate, revealing the intriguing story of Mars's water-rich past.

Maria T. Zuber is in the Department of Earth, Atmospheric and Planetary Sciences, Massachusetts Institute of Technology, Cambridge, Massachusetts 02139, USA. e-mail:zuber@mit.edu

1. Parker, T. J., Gorsline, D. S., Saunders, R. S., Pieri, D. C. \& Schneeberger, D. M. J. Geophys. Res. Planets 98, 11061-11078 (1993).

2. Malin, M. C. \& Edgett, K. S. Geophys. Res. Lett. 26, 3049-3052 (1999).

3. Citron, R. I., Manga, M. \& Hemingway, D. J. Nature 555, 643-646 (2018).

4. Bouley, S. et al. Nature 531, 344-347 (2016).

5. Carr, M. H. Water on Mars 197 (Oxford Univ. Press, 1996).

6. Baker, V. R. et al. Nature 352, 589-594 (1991).

7. Head, J. W. III et al. Science 286, 2134-2137 (1999).

8. Mouginot, J., Pommerol, A., Beck, P., Kofman, W. \& Clifford, S. M. Geophys. Res. Lett. 39, L02202 (2012).

9. Villanueva, G. L. et al. Science $\mathbf{3 4 8 , 2 1 8 - 2 2 1}$ (2015).

10.Perron, J. T., Mitrovica, J. X., Manga, M., Matsuyama, I. \& Richards, M. A. Nature 447, 840-843 (2007).

11.Goldreich, P. \& Toomre, A. J. Geophys. Res. 74, 2555-2567 (1969).

12. Roberts, J. H. \& Zhong, S. Icarus 190, 24-31 (2007).

13. Matsuyama, I. \& Manga, M. J. Geophys. Res. Planets 115, E12020 (2010)

14.Clifford, S. M. \& Parker, T. J. Icarus 154, 40-79 (2001)

15.Carr, M. H. \& Head, J. W. III J. Geophys. Res. Planets 108, 5042 (2003).
SENSORY SYSTEMS

\section{A trio of ion channels takes the heat}

Of the various temperature-sensitive ion channels identified previously, three have now been found to act in concert to detect painful heat and initiate protective reflexes. SEE LETTER P.662

\section{ROSE Z. HILL \& DIANA M. BAUTISTA}

$\mathrm{T}$ There's nothing cosier than sitting by the fire on a cold night, and mammals have an amazing ability to find the perfect spot in which to warm themselves without being burnt. This ability is mediated by specialized heat-sensitive neurons of the somatosensory system that innervate the skin. These neurons are fine-tuned to detect the temperature at which pleasurable warmth turns into painful (noxious) heat, and are responsible for initiating protective reflexes and triggering avoidance behaviours. The search for temperature sensors in somatosensory neurons has uncovered several ion channels that are part of the transient receptor potential (TRP) family ${ }^{1-4}$, but the TRP channel or channels that are responsible for heat-evoked pain have remained unclear. Vandewauw et al. ${ }^{5}$ report on page 662 that three TRP channels work together to detect noxious heat in mice.

The first temperature-sensitive TRP channel, TRPV1, was discovered more than 20 years ago ${ }^{1}$. TRPV1 has a crucial role in the development of hypersensitivity to heat after injury or inflammation, but experiments have revealed that mice that lack TRPV1 display only a partial defect in the ability to sense and respond to noxious heat ${ }^{6}$. Another TRP channel in somatosensory neurons, TRPM3, is also activated in response to painful temperatures; however, heat-evoked pain-avoidance behaviours are only partially attenuated in mice that lack this channel ${ }^{3}$, leaving the mystery of how painful heat is sensed unsolved.

Vandewauw et al. report that double-mutant mice, which lack both the TRPV1 and TRPM3 channels, show only mild defects in heatevoked pain-avoidance behaviours, similar to mice that lack just one of those channels. By contrast, they find that mice that lack a third channel, TRPA1, as well as TRPV1 and TRPM3, do not display any protective avoidance behaviours when exposed to noxious heat (Fig. 1). The three channels therefore act in concert to mediate behavioural responses to noxious heat.

What led Vandewauw and colleagues to implicate TRPA1 in heat sensing? The authors observed that a subset of the heat-responsive somatosensory neurons that normally express TRPV1 and TRPM3 also express TRPA1, which has previously been shown to mediate inflammatory pain $^{7}$ and itching ${ }^{8}$ in mice. TRPA1 is known to contribute to the heatsensing abilities of non-mammalian organisms, including rattlesnakes ${ }^{9}$ and fruit flies ${ }^{10}$, but mice that lack TRPA1 exhibit normal
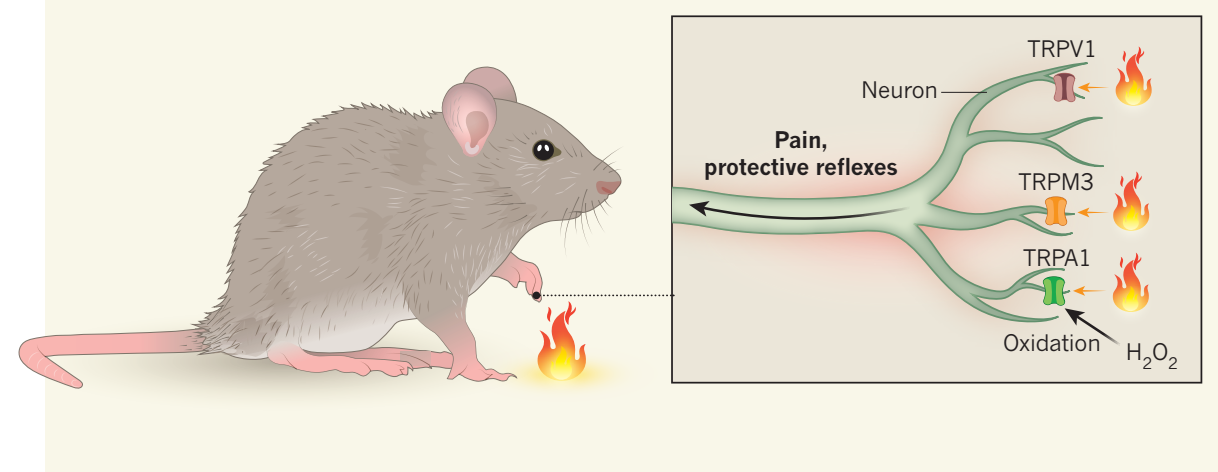

Figure 1 | Three heat-sensitive TRP channels. Vandewauw et al. ${ }^{5}$ demonstrate in mice that potentially damaging temperatures excite pain-sensing neurons of the peripheral nervous system through the activity of three ion channels from the TRP family - TRPV1, TRPM3 and TRPA1. Activation of TRPA1 (by, for example, hydrogen peroxide, $\mathrm{H}_{2} \mathrm{O}_{2}$ ) is likely to require oxidation of certain amino-acid residues, as well as the presence of heat. Activation of the three channels causes neuronal firing and the transmission of signals to the central nervous system, leading to pain-associated behaviours and protective reflexes. 
avoidance of noxious heat ${ }^{7,11}$. Therefore, the protein has not been implicated in heat sensing in mammals - until now.

Activation of this channel by heat in rattlesnakes or fruit flies involves specialized sequences of amino acids known as ankyrin repeats at the amino terminus ${ }^{12}$. These repetitive stretches are different in mammalian TRPA1, making the channel insensitive to heat under normal conditions ${ }^{12}$. By contrast, activation of TRPA 1 in mice by inflammatory molecules is dependent on the oxidation state of three cysteine amino-acid residues located adjacent to the ankyrin repeats ${ }^{13}$. Vandewauw and colleagues therefore proposed that oxidation of these residues might be sufficient to activate the heat-sensing capabilities of mouse TRPA1.

To trigger oxidation of TRPA 1 in vitro, the authors applied hydrogen peroxide to sensory neurons that lacked TRPV1 and TRPM3 but expressed TRPA1. They found that hydrogen peroxide treatment did sensitize TRPA1 in such a way that heat could now excite the neurons. This suggests that mouse TRPA 1 must be oxidized to respond to heat. However, the authors do not provide a physiological mechanism for how TRPA1 might be oxidized in vivo to participate in heat sensing. This will no doubt be an area for future investigation.

Vandewauw and colleagues also performed electrophysiological experiments, which revealed that the three TRP channels they studied are co-expressed in more than one class of somatosensory nerve fibre - including at least two types of pain-sensing neuron that encode distinct sensations, such as dull pain or sharp pain. The expression of these channels in different neuronal subtypes might give rise to complex circuitry and crosstalk that aids the rapid avoidance of noxious heat. In addition, it could add a further layer of redundancy to an important protective response.

Finally, the group showed that, although the triple-mutant mice do not exhibit protective heat-avoidance reflexes, they do have the same preference as control mice for innocuous warm temperatures $\left(30^{\circ} \mathrm{C}\right)$ over noxious high temperatures $\left(45^{\circ} \mathrm{C}\right)$ when presented with a choice. This finding suggests that the sensing of painful heat might not have a substantial role in determining a preference for pleasurable temperatures over painful ones, warranting further investigation of warmth sensing - an area of active debate. A previous study has shown that whereas heat-responsive spinal neurons, which receive input from heat-sensitive somatosensory neurons, respond to absolute temperatures, cold-responsive spinal neurons, which receive input from coldsensitive somatosensory neurons, respond to relative changes in temperature ${ }^{14}$. Together, these data imply that the main role of peripheral heat-sensing neurons, such as those described by Vandewauw et al., might be to mediate avoidance of high temperatures, rather than to set an animal's preferred temperature range.

Circuit-tracing and in vivo imaging will no doubt reveal the contributions of different subsets of peripheral neurons to the various representations of cold, cool, warm and hot temperatures in the central nervous system. Decoding these circuits will help to unravel the nuances of sensory perception and to elucidate the basis for diverse temperature preference between species or individual organisms.

Rose Z. Hill and Diana M. Bautista are in the Department of Molecular and Cell Biology, University of California, Berkeley, California 94720, USA. D.M.B. is also at the Helen Wills Neuroscience Institute, University of California, Berkeley. e-mails: rzhill@berkeley.edu; dbautista@berkeley.edu

1. Caterina, M. J. et al. Nature 389, 816-824 (1997)

2. Patapoutian, A., Peier, A. M., Story, G. M. \& Viswanath, V. Nature Rev. Neurosci. 4, 529-539 (2003).

3. Vriens, J et al Neuron 70, 482-494 (2011)

4. Tan, C. H. \& McNaughton, P. A. Nature 536, 460-463 (2016).

5. Vandewauw, I. et al. Nature 555, 662-666 (2018)

6. Caterina, M. J. et al. Science 288, 306-313 (2000).

7. Bautista, D. M. et al. Cell 124, 1269-1282 (2006).

8. Wilson, S. R. et al. Nature Neurosci. 14, 595-602 (2011).

9. Gracheva, E. O. et al. Nature 464, 1006-1011 (2010)

10.Neely, G. G. et al. PLoS ONE 6, e24343 (2011).

11.Kwan, K. Y. et al. Neuron 50, 277-289 (2006)

12.Cordero-Morales, J. F., Gracheva, E. O. \&

Julius, D. Proc. Natl Acad. Sci. USA 108,

E1184-E1191 (2011)

13. Macpherson, L. J. et al. Nature 445, 541-545 (2007)

14.Ran, C., Hoon, M. A. \& Chen, X. Nature Neurosci. 19, 1201-1209 (2016).

This article was published online on 14 March 2018.

\section{CHEMISTRY}

\section{AI designs organic syntheses}

Software that devises effective schemes for synthetic chemistry has depended on the input of rules from researchers. A system is now reported in which an artificial-intelligence program learns the rules for itself. SEE ARTICLE P.604

\section{DEREK LOWE}

S ynthetic organic chemistry is the science of building desired chemical structures from simpler parts. The knowledge and experience of researchers has always been the key to combining chemical reactions into successful synthetic schemes. But on page 604 , Segler et al. ${ }^{1}$ report that an artificialintelligence program can design routes for synthesizing compounds that - at least, on paper - seem just as good as those produced by humans.

Organic chemists often work by thinking backwards as much as they do forwards when designing a synthetic route. The concept of retrosynthesis ${ }^{2}$, introduced by E. J. Corey in the 1960s, and for which he was awarded the Nobel Prize in Chemistry in 1990, codified the way in which many chemists think (Fig. 1). When looking at a target molecule, they ask: "What could this have been made from? Which bonds could have been formed, and which atoms or chemical groups could have been added or transformed?" Then, the process starts again, as researchers try to determine the reactions that could have led to the precursor molecule. The aim is to work back to easily available starting compounds, while balancing the factors that make a good synthesis - including the number of steps involved, the probable product yields of those steps, and the ease of use of the chemistry involved. Organic chemists deal constantly with such questions, for example when making compounds for testing in drug-discovery programmes.

Since the birth of synthetic organic chemistry in the mid-nineteenth century, a huge number of synthetic organic reactions have been reported across a literature that gets larger every hour. Before the 1980s, many chemists kept collections of handwritten, cross-referenced index cards containing useful reactions from the literature, to guide the design of synthetic pathways. These aidememoires moved naturally on to digital databases as computer technology became widespread.

These days, chemists review the various methods for turning chemical group $\mathrm{X}$ into chemical group Y by drawing the molecular structures of interest using a computer program and then performing an online search for relevant reactions. This almost invariably produces a long list, from which researchers must select the most appropriate reaction for their needs, according to their knowledge and experience. Stringing such reactions into a useful 